\title{
Quark-hunters are rewarded
}

\section{Washington}

THE Nobel physics prize committee this year took what may be the final step in its curiously unchronological recognition of the experimental discoveries that have established, over the past few decades, the generally accepted standard model of elementary particle physics. The winners, Jerome Friedman and Henry Kendall of the Massachusetts Institute of Technology and Richard Taylor of the Stanford Linear Accelerator Center (SLAC), collaborated in 1967 on electron-proton scattering studies at SLAC that demonstrated the existence of smaller particles inside the proton. Murray Gell-Mann, a co-inventor with George Zweig of these subparticles, named quarks, won the physics prize in 1969 , but, for reasons the secretive Swedes are unlikely to reveal, it has taken another 21 years for the experimental work that substantiated Gell-Mann's hypothesis to be similarly rewarded.

Although the results obtained by Friedman, Kendall and Taylor turned out to be of fundamental importance, their experiment was originally seen as a longshot at best, a waste of accelerator time at worst. When it came on-line in 1966, the Stanford Linear Accelerator was mostly devoted to studies of elastic electron scattering, in which the aim was to record the deflections and energy losses of electrons bounced off protons. Inelastic scattering experiments, which Friedman, Kendall and Taylor decided to pursue, were referred to by some as "beam surveys": an electron was slammed into the protons at high energy to create a shower of new particles, and the debris was examined to see if any useful secondary beams (of muons, for example) could be extracted.

The standard wisdom at the time was that the mess of debris created by inelastic scattering was too complex to be understood in a way that would shed light on the inner structure, if there were any, of the proton. Elastic scattering, the modern version of Rutherford's famous experiment in which he scattered alpha particles off atoms to show the presence of small, dense, electrically charged atomic nuclei, was instead thought to be the way to probe the proton. But the first round of results from elastic scattering experiments showed nothing of interest, indicating only that the proton seemed to be a smooth, structureless distribution of charge. In retrospect, this was inevitable. Elastic scattering could have picked up the existence of a hard core to the proton, as in the Rutherford experiment, but charged quarks moving rapidly about inside the proton would, to the passing electron, be indistinguishable from a smooth charge distribution.
Inelastic scattering produced, as expected, a complex mess of new particles, but Friedman, Kendall and Taylor found that the statistical properties of this mess behaved in a relatively simple way at higher energies. This 'scaling' of the results of deep inelastic scattering (deep because the more energetic electrons were able to penetrate further into the proton) did not have any immediate interpretation, but it struck some theoretical physicists, in particular James Bjorken and Richard Feynmann, as a clue to some underlying simplicity in proton structure.

Feynmann realized that scaling made sense if, at high energies, the electrons were interacting with individual subunits of the proton rather than with its constituents as a whole. He called the subunits partons, and argued that the results of deep inelastic scattering showed that the partons moved rather freely inside the protons. This seemed to be at odds with Gell-Mann's theory, because his quarks had to be strongly interacting. At the same time, according to Bjorken, there were doubts about the reality of the scaling laws, and also alternative explanations for them that involved not proton substructure but new dynamical effects in the electron-proton interaction.

The programme of deep inelastic scattering studies was to continue for many years, accumulating results that allowed different explanations to be distinguished. On the theoretical front it was eventually realized that, at sufficiently high energies, quarks could theoretically behave at close range as if they were free particles, but still be inextricably bound up inside the proton.

By the mid-1970s, quarks had become real particles, not abstractions. And in the long run the success of the quark model put physics on the road towards unification mania, the goal of which is to assemble all the forces of nature into some single, allencompassing model.

This year's physics award, in conjunction with that of two years ago, when Leon Lederman, Melvin Schwartz and Jack Steinberger were made Nobel laureates for their discovery of neutrinos in the early 1960s, corrects an omission that elementary particle physicists had long noted. In contrast, the 1983 discovery of the $\mathrm{W}$ and $\mathrm{Z}$ particles, crucial to unification of the weak and electromagnetic forces, was promptly recognized when Carlo Rubbia and Simon van der Meer won the physics prize the following year.

With most notable particle discoveries now rewarded, it may be that physicists will have to find the top quark or the electroweak Higgs boson before the Nobel will come their way again.

David Lindley
Corey the
logical choice

\section{London}

THIS year's Nobel prize in chemistry has been awarded to Elias Corey of Harvard University, Massachusetts, for his contributions to synthetic organic chemistry. Corey is widely credited by chemists as having played a key part in transforming organic synthesis from something resembling a 'black art' to a discipline founded firmly on logic. That he has won the prize alone rather than sharing it is itself a testament to his position in the field and the pervasive influence of his ideas.

Over the past three decades, Corey has devised synthetic routes to more than 100 natural products, many of which have found wide use in medicine and industry. $\mathrm{He}$ is perhaps best known for leading the group that in 1969 made the first synthetic prostaglandins, molecules involved in the regulation of among other things blood pressure and the heart. But it was not so much his prolific output that attracted the Nobel Committee as his strictly logical approach to complex syntheses.

In the $1950 \mathrm{~s}$, at the start of his career, organic synthesis was largely a trialand-error pursuit. Progress was being made in understanding the mechanisms of organic reactions and in developing useful reagents, but synthetic chemists still analysed target molecules case by case.

In the absence of general rules, the prevailing approach was to identify a possible starting subunit (a readily available reagent, for example) within the structure of the target molecule. The problem then became how to manipulate the subunit so as to generate the full structure.

This approach led to some notable successes, such as Robert Woodward's heroic synthesis of chlorophyll, one of the achievements that earned him the Nobel prize in chemistry in 1965 . Yet by and large, chemical manipulations were still deployed in an ad hoc fashion, a practice that remained the norm until the $1960 \mathrm{~s}$ when Corey began working on sesquiterpenes, a family of natural products whose structures were void of obvious starting subunits.

Corey reasoned that to devise logical synthetic routes to such molecules, it was necessary to work backwards from the end product, disconnecting chemical structure until one obtained a set of simple precursors. In seminal papers published in 1967 and 1968, Corey wove his ideas into a general synthetic strategy, coining the term 'retrosynthetic' analysis to describe it.

Put crudely, the analysis involves subjecting the target molecule of the synthesis to a series of stepwise dissections, according to a set of simple rules bearing on the 